\title{
Robust Wireless Video Streaming Using Hybrid Spatial/Temporal Retransmission
}

\author{
Mei-Hsuan Lu, Student Member, IEEE, Peter Steenkiste, Fellow, IEEE, and Tsuhan Chen, Fellow, IEEE
}

\begin{abstract}
Bandwidth demands and timing constraints are two major challenges for wireless video streaming applications. In this paper, we present a hybrid spatial/temporal retransmission protocol that tackles both of these challenges. To increase individual throughput as well as overall network capacity, the system uses an opportunistic retransmission protocol (PRO, Protocol for Retransmitting Opportunistically) that relies on overhearing nodes distributed in physical space to function as relays that retransmit failed packets on behalf of the source. Specifically, the best relay out of the set of nodes that currently have the copy of the packet is responsible for retransmitting (relaying) the packet. Relays with stronger connectivity to the destination have a higher chance of delivering packets successfully than the source, thereby resulting in a more efficient use of the channel. To meet timing constraints, a Time-based Adaptive Retransmission strategy (TAR) is applied by both the source and the relays. With TAR, the MAC dynamically determines whether to (re)transmit or discard a packet based on the retransmission deadline of the packet assigned by the video server. This significantly reduces the number of late packet arrivals at the receiver. Our extensive evaluation results both on a testbed and in the real world demonstrate that hybrid temporal/spatial retransmission can boost streaming performance in diverse wireless environments. The benefits are most pronounced for busy networks, under fading conditions, or for mobile users.
\end{abstract}

Index Terms - Wireless video streaming, time-based retransmission, opportunistic retransmission, 802.11 WLAN.

\section{INTRODUCTION}

W ITH the wide adoption of wireless LANs, wireless multimedia applications have received a lot of attention. However, wireless video transmission poses many challenges, including coping with high bandwidth demands and strict timing constraints. In this paper, we present a hybrid spatial/temporal retransmission protocol, building on our earlier work in [16] and [13], to tackle these two challenges. To increase individual throughput as well as overall network capacity, the system runs an opportunistic retransmission protocol (PRO, Protocol for Retransmitting Opportunistically) that relies on overhearing nodes, if any, distributed in physical space to function as relays that retransmit failed packets on behalf of the source [16]. Specifically, the best relay out of the set of nodes that currently have the copy of the packet

Manuscript received 1 March 2009; revised 1 November 2009. This work was supported by NSF award CCR-0205266 and by the iCAST project.

Mei-Hsuan Lu is with the Department of Electrical and Computer Engineering, Carnegie Mellon University, Pittsburgh PA 15213-3890 (e-mail: amylu@cmu.edu).

Peter Steenkiste is with the School of Computer Science and Department of Electrical and Computer Engineering, Carnegie Mellon University, Pittsburgh PA 15213-3890 (e-mail: prs@cs.cmu.edu).

Tsuhan Chen is with the School of Electrical and Computer Engineering, Cornell University, Ithaca NY 14850-2432 (e-mail: tsuhan@ece.cornell.edu).

Digital Object Identifier 10.1109/JSAC.2010.100418. is responsible for retransmitting (relaying) the packet. Relays with stronger connectivity to the destination have a higher chance of delivering packets successfully than the source, thereby resulting in a more efficient use of the channel. Compared with traditional relaying-based schemes that set up long-term paths, PRO takes advantage of packet reception outcomes that are inherently random and unpredictable, which leads to higher efficiency and greater agility in combating dynamic channel fading and shadowing.

To fulfill timing constraints, we apply our prior work of Time-based Adaptive Retransmission (TAR) on both the source and the relays [13][15]. With TAR, the MAC dynamically determines whether to (re)transmit or discard a packet based on the retransmission deadline of the packet assigned by the video server, regardless of how many times the packet has been transmitted. Unlike 802.11-like retransmission strategies that adopt a fixed retry limit, TAR dynamically adapts the maximum number of transmissions based on current channel conditions and media characteristics. This significantly reduces the number of late packets [6].

The proposed hybrid spatial/temporal retransmission scheme, called PROTAR, draws on the combined strength of TAR and PRO [14]. Combining TAR and PRO is however a nontrivial task. One challenge is that PROTAR needs consistent use of deadlines across multiple relays, but the clock of relays may not be synchronized. The coordination must have low overhead so the gain of time-based relaying is not compromised.

The key contributions of this paper are as follows. First, we present a simple and efficient design for PROTAR to improve wireless video transmission. Second, we describe how we implemented PROTAR in the Madwifi driver for wireless NICs based on the Atheros chipset. Third, we derive an analytical model for PROTAR building on new models for PRO and TAR. We also provide numerical results based on the analysis to quantify the gains that are theoretically achievable by PROTAR. Finally, we evaluate PROTAR using both PSNRbased measurement studies and extensive user studies on both a controlled testbed and in the real world. Our extensive experimental results demonstrate that the adoption of PROTAR can improve the quality of video streaming in many wireless LANs.

The rest of this paper is organized as follows. Section II discusses related work in the area of wireless video streaming. Section III presents a short review of PRO and TAR and then describes how they can be integrated to optimize video streaming. Section IV elaborates on the design and implementation of PROTAR. A probabilistic analysis for TAR, PRO, 
and PROTAR are provided in Section V. Section VI presents experimental evaluation of PROTAR based on both objective and subjective visual test results. Section VII summarizes the paper.

\section{RELATED WORK}

Many solutions have been proposed to improve the quality of wireless video streaming. One approach is to apply additional controls in the application layer at the video source [1], [2]. Other approaches rely on cross-layer optimization consisting of protection strategies implemented in the protocol stacks on the end systems [3]. These end-to-end techniques require no support from the network, but may not react promptly in dynamic wireless network conditions.

More recently researchers have focused on adding intelligence to the network as a means of improving wireless video streaming. Compared with end-to-end approaches, these schemes are more agile in reacting to network dynamics. In [6] and [9], different retry limits are associated with different priorities in the radio link layer of cellular networks. Important frames are given a higher priority so they are granted more retransmission attempts. In [4], Li and van der Schaar propose a real-time retry limit adaptation algorithm that traces the optimal MAC-layer retry limit over wireless LANs. The proposed scheme also provides unequal error protection for layered video streams by using different retry limits. Bucciol et al. in [11] propose a scheduling metric that jointly considers perceptual and temporal importance. This metric is then used by the link layer to drive the packet-selection process for each retransmission opportunity. In [5], Liebl et al. propose deadline-aware scheduling of video streams over a wireless shared channel. By incorporating information about the video stream structure and future channel behavior, the proposed algorithm can achieve a fairer distribution of video quality among all users. Another active research area is rate-distortion optimized scheduling based on [7]. Like prior work, PROTAR is a local time-based retransmission mechanism that requires support in the network. The main difference is that PROTAR adopts a simple FIFO queueing discipline, which can be easily implemented in the link layer.

The contributions discussed so far focused on video transmission over a single path. To provide robustness against errors, path diversity has emerged as an appealing solution. The fundamental hypothesis underlying path diversity is that in wireless networks, errors are often path or location dependent, so the use of multiple paths increases the chances of successful packet delivery. In [8], Miu et al. propose to use multiple paths simultaneously or to switch between them (site selection) based on channel characteristics in order to achieve low-latency video transmission in an infrastructure wireless LAN. In [12], Li et al. propose a multi-source video streaming system to support high quality video streaming over IEEE 802.11-based wireless networks. The idea is to leverage nearby wireless peers by having them form a joint sender group with the remote media server. Path diversity has also been adopted in wireless cellular/WLAN standards. For instance, the $802.16 \mathrm{j}$ specification adopts cooperative transmission, a mesh networking-based scheme that can improve node connectivity [27]. HSDPA uses opportunistic scheduling

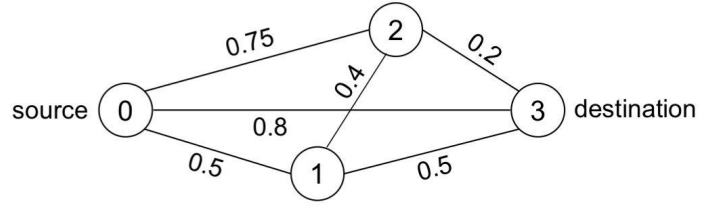

Fig. 1. A four-node network with link error rates shown along the edges of the graph. In this network, node 0 is the source; node 3 is the destination; node 1 and node 2 are candidate relays.

that adapts link-layer transmission based on channel quality indicator (CQI) reports from user equipments (UE) to increase efficiency [28]. Similar ideas have also been used in multi-hop wireless routing. In [33], opportunistic routing improves the performance of static predetermined routes by determining the route as the packet moves through the network based on which nodes receive each transmission.

PROTAR also leverages path diversity to increase the probability of successful transmissions. However, it differs from prior work in several aspects. Unlike existing linklayer relaying methods that rely on long-term paths, PROTAR offers dynamic packet traversal on a per-packet basis based on the opportunistic receptions at relays. This property makes PROTAR extremely useful in combating wireless channel shadowing and fading. Moreover, PROTAR automatically avoids routing overheads. Finally, PROTAR does not increase (or may even decrease) packet latency or reorder packets, while opportunistic routing often does increase latency and generate out-of-order deliveries in order to spread out scheduling and routing overheads, which is a problem for interactive applications.

\section{HYBRID SPATIAL/TEMPORAL RETRANSMISSION}

In this section, we first review PRO and TAR and we then describe how they can be integrated to optimize video transmission.

PRO leverages the fact that, in the wireless environment, broadcast is free (from the sender's perspective) and that errors are mostly location dependent [16]. Hence, if the intended recipient does not receive the packet, other nodes may have overheard the packet so they have become a candidate sender for that packet. With multiple candidate senders distributed in space, the chance that at least one of these available senders has a good channel to the destination is increased. Consider the network in Fig. 1. Node 0 is the source and node 3 is the destination. Due to the broadcast nature of the wireless medium, transmissions from node 0 to node 3 may be overheard by node 1 and node 2 . When the packet transmission to node 3 fails, but the packet is overheard by node 1 , then it would be beneficial to have node 1 retransmit on behalf of node 0 , because node 1 has a higher chance of successfully delivering the packet than node 0 . The same scenario also applies when node 2 overheard the packet. When both nodes overheard the packet, node 2 is a more suitable relay than node 1 . PRO takes advantage of packet reception outcomes that are inherently random and unpredictable by postponing the selection of a relay until after the original transmission completes. This allows PRO to dynamically select the best node to retransmit each packet, while conventional relaying methods select a longer term path based on average performance [8], [12], [27]. 
For video applications, retransmission of late packets by either sources or relays not only wastes bandwidth but it can delay later packets, potentially making them late, resulting in serious degradation of visual quality [10]. TAR is a linklayer strategy that directly tackles this issue [13][15]. Unlike the 802.11 MAC which discards a packet when a predefined, fixed retry limit is reached, a TAR-enabled MAC adaptively decides whether to discard or (re)send a packet based on a retransmission deadline associated with the packet. The retransmission deadline is assigned by the application according to the application's specific requirements. This strategy allows the MAC to promptly respond to changes in network conditions and traffic characteristics.

PROTAR combines the strengths of both TAR and PRO [14]. The source, as specified in TAR, transmits a packet based on the retransmission deadline associated with the packet. Upon a failed transmission, if the retransmission deadline has not expired, either one of the relays that overheard the packet or the source will retransmit the packet, depending in part on current channel conditions. This time-based opportunistic retransmission process continues until either the packet is delivered successfully or the retransmission deadline has passed. If no relay is present or willing to participate, PROTAR automatically falls back to TAR.

\section{DESIGN AND IMPLEMENTATION OF PROTAR}

While the ideas underlying PROTAR are simple, turning these ideas into a practical system is not easy. In this section, we elaborate on the design and implementation of PROTAR. Fig. 2 shows the system diagram. The design of PRO for regular data traffic can be found in [17]. Here we focus on the key components that directly relate to the operation of PROTAR.

\section{A. Estimating Link Quality and Selecting Participating Relays}

Using poor relays can hurt performance because it offers little opportunistic gain while increasing the consensus overhead. PROTAR requires an effective and efficient measure of link quality to decide whether a node is a suitable relay. To this end, we use the Received Signal Strength Indicator (RSSI), available on most commodity wireless hardware, to estimate instantaneous link quality. This is motivated in part by recent work, e.g. [19], that shows that RSSI is generally a nearly linear function of the actual received signal strength. Relays listen to packets from the source and the destination to create an RSSI history for each node. The RSSI history is then used to compute a time-weighted average RSSI. If this average for a particular destination is above a threshold, then the node becomes a qualified relay for that destination. Qualified relays share their link quality information via periodic broadcasts and based on that, decide whether to participate in the retransmission. The resulting participating relays are selected such that the probability of overhearing the source by one of them is high while the consensus overhead is low. Note that sources always participate in the retransmission after a failed initial transmission.

\section{B. Relay Prioritization}

When multiple participating relays overheard a failed transmission, we need to prioritize the relays, so the relay with the best channel to the destination is most likely to retransmit the packet. Centralized solutions are not attractive because they involve extra overhead in scheduling feedback distribution and extra bandwidth in distributing feedback. To reduce overhead, PROTAR adopts a distributed method for relay prioritization by leveraging the 802.11 random backoff process.

The 802.11 standard requires that each sending station backs off for a randomly selected period of time before transmitting a packet [21]. The backoff period is uniformly distributed in a range, called the contention window. For collision resolution, the size of contention window doubles after a failed transmission until a maximum size is reached. PROTAR leverages the 802.11 backoff process to prioritize relays. Specifically, high priority relays are assigned a smaller contention window than lower priority relays. The priority of a relay is determined based on the rank of the quality of its channel to the destination, relative to the other participating relays. Relays associated with a small contention window contend for the channel with a shorter backoff interval so they are more likely to transmit first.

\section{Retransmitting Packets}

Relays detect failed transmissions through the lack of an ACK. Participating relays contend to retransmit the packet similar to retransmitting a local packet. Relays terminate the retransmission process in response to the following events:

- The retransmission deadline of the packet has elapsed. We will describe how relays retrieve the retransmission deadline later.

- An ACK frame destined for the source is overheard. This implies successful reception.

- A new data packet (i.e. the packet stamped with a larger sequence control number) originating from the source is overheard. This means either that the source has discarded the current packet, or that the packet was successfully delivered but this relay missed the ACK. In either case, the relay should stop retransmitting the packet.

When a relay misses an ACK, it may send a duplicate transmission. In practice this is rare since ACKs are short packets that are transmitted using the base transit rate, so they are more robust against channel erasures. In [17], a method that effectively relays ACKs to suppress duplicate transmissions is presented.

\section{Retrieving the Retransmission Deadline}

To enable time-based relaying, the packet deadlines need to be interpreted consistently across relays. We now describe a simple protocol that eliminates the need for clock synchronization among relays. We introduce a new MAC header field, namely time_to_relay, appended to the standard 802.11 packet header. The time_to_relay field is initialized by the source and updated by relays to reflect how much time remains before a packet should be dropped. The source node initiates this field based on the following rule:

$$
\text { time_to_relay }=D-T
$$




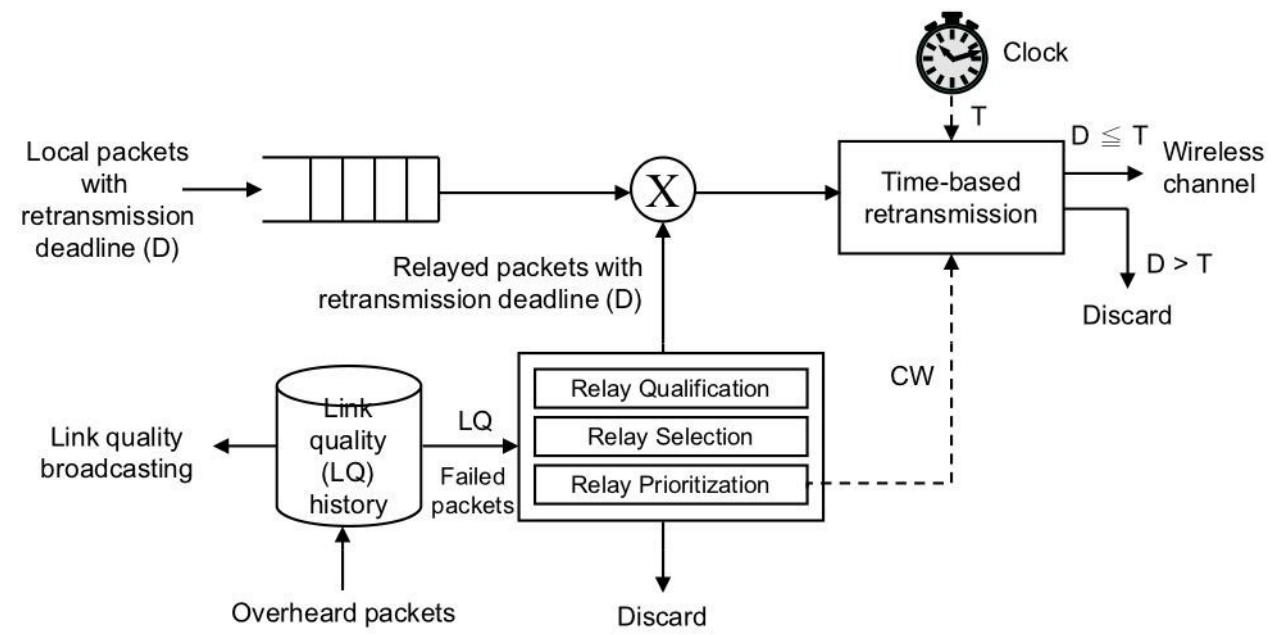

Fig. 2. System diagram of PROTAR

where $D$ is the retransmission deadline and $T$ is the time that the packet is sent on the air. When a relay overhears a packet, it reconstructs the retransmission deadline of that packet using:

$$
D=R+\text { time_to_relay }- \text { duration }
$$

where $R$ is the packet receive time based on the local clock, and duration is the value of time needed to transmit the packet which is carried in the packet header.

Relays may retransmit a packet as long as the retransmission deadline is larger than the current time. Before sending a packet, relays update the time_to_relay field based on (1) so that receivers of the packet can correctly reconstruct the retransmission deadline.

\section{E. A Note on Protocol Overhead}

PROTAR involves two sources of overheads: the time_to_relay field in each data packet and periodic broadcast messages. The time_to_relay field reduces the maximum data length by 2 to 2310 bytes. The resulting overhead is small since video is often packetized into large MAC frames. The broadcast messages contain averaged RSSI values for the sources and destinations that a relay is qualified to relay for. The resulting overhead is linear with the number of qualified relays and source-destination pairs in service but it is relatively small as compared to data packets. The periodic broadcast frequency is 1 second in our implementation. This value is borrowed from the default HELLO message interval used in AODV [31]. Relays can further reduce the broadcast overhead by adapting the broadcast frequency based on how fast the channel conditions change. They can also suppress broadcasts when the chance of becoming an eligible relay is low.

Table I summarizes the parameters used in our implementation. Readers may refer to [17] for more details.

\section{AnALYsis}

In this section, we analyze the performance of TAR, PRO, and PROTAR. We first show that TAR adaptively achieves the best retransmission strategy without the requirement of channel information and traffic statistics. We then give an analytical model that quantifies the performance of PRO. Finally,
TABLE I

PROTOCOL PARAMETERS USED IN THE CURRENT IMPLEMENTATION $(\dagger$ THIS IS THE DEFAULT VALUE. THIS THRESHOLD IS CALIBRATED ONLINE.)

\begin{tabular}{l|l}
\hline Parameter & Value \\
\hline Relay Qualification RSSI Threshold & $10 \mathrm{~dB} \dagger$ \\
\hline Relay Selection Probability Threshold & 0.9 \\
\hline $\mathrm{CW}_{\min }$ of the highest two relays & 32 slots \\
\hline $\mathrm{CW}_{\min }$ of the next highest two relays & 64 slots \\
\hline $\mathrm{CW}_{\min }$ of the rest relays & 128 slots \\
\hline Link quality broadcast interval & $1 \mathrm{sec}$ \\
\hline
\end{tabular}

we present analytical results for PROTAR by combining the analysis of TAR and PRO.

\section{A. Analysis of Time-based Adaptive Retransmission}

When streaming video over wireless channels, there are two causes for packet loss: link packet erasures (i.e., dropped by senders after too many retries), and late arrivals (i.e., missed transmission deadlines due to incurred delay). Late arrivals can be mitigated by introducing long startup delay at the receiver. However, this may not work for video streams with hard latency constraints (e.g. interactive video) or when the receiver has limited buffer space. To simplify the analysis, we assume that video is generated at a constant frame rate and that the deadline for each video frame is one inter-frame interval after that frame is generated. We further assume that each video frame is transmitted as a single MAC packet with a fixed transmission time. Let $\lambda$ be the packet arrival rate (packets/seconds). The packet arrival time of packet $i$ can be written as $A(i)=i / \lambda$ and the deadline of packet $i$ is $D(i)=(i+1) / \lambda$. Let $\mu$ be the service rate of the link (packets/second) and $P_{e}$ be the link error rate. If we assume that the wireless link is a memoryless packet erasure channel such that packets losses are independent with a constant probability, then the number of transmissions for successfully delivering a packet is a geometric random variable:

$$
s_{k}^{(T A R)}=\left(1-P_{e}\right) P_{e}^{k-1} .
$$

In TAR, packet $i$ is discarded when the sender cannot deliver it before the deadline $D(i)$ so there are no late arrivals at the destination. This also implies that the transmission of packet $i+1$ will start at the time it enters the system, independent 
of the transmission outcomes of the previous packets. Given constant packet arrival and link service rates, the number of transmissions that must occur before a packet misses the deadline is then $\lfloor\mu / \lambda\rfloor$. Thus the packet loss probability of TAR, occurring when the workload of retransmissions exceeds the link capacity, can be calculated as

$$
p_{\text {loss }}^{(T A R)}=\sum_{k=\lfloor\mu / \lambda\rfloor+1}^{\infty} s_{k}^{(T A R)}=P_{e}^{\left\lfloor\frac{\mu}{\lambda}\right\rfloor} .
$$

For 802.11-like retransmission strategies, packets are retransmitted until a fixed retry limit $R$ is reached regardless of the deadline associated with the packet. The probability distribution of the number of transmissions for a packet can thus be written as

$$
s_{k}^{(802.11)}= \begin{cases}\left(1-P_{e}\right) P_{e}^{k-1} & \text { if } 1 \leq k \leq R \\ P_{e}^{R} & \text { if } k=R+1 \\ 0 & \text { otherwise }\end{cases}
$$

This indicates that packet losses are caused by either packet erasures (the retry limit is too small) or late arrivals (the retry limit is too large). The link packet erasure probability (i.e. the packet drop rate after $R$ unsuccessful retries) is given by

$$
p_{\text {error }}^{(802.11)}=P_{e}^{R+1} \text {. }
$$

The probability of late arrivals is more difficult to calculate. Let us consider a newly arrived packet $i$. The packet can face one of two situations: (A) the transmission of packet $i-1$ has already finished (either successfully delivered or discarded after the retry limit is reached) so the system can immediately start the transmission of packet $i$; (B) the system must delay the transmission of packet $i$ until the transmission of packet $i-1$ is completed. This means that the transmission outcome of a packet is now dependent on that of the previous packets.

Analyzing a system with memory is generally difficult. Fortunately, the memory structure of the system is statistically describable. Every time the system finishes the transmission of a packet before the deadline, transmissions of future packets are independent of the past (i.e. transmission of the next packet will start at the packet arrival time). This means that we can simply study the system behavior between two consecutive packets whose transmissions are finished before their deadlines. In other words, we study the statistics of a burst of late packets.

Let $Z_{i}$ denote the number of transmissions for packet $i$, which is an i.i.d process with probability distribution given in (5). Call packet $i$ a valid packet if its transmission is finished before $D(i)$; otherwise it is a late packet. Consider, for now, that packet 0 is a valid packet. Then the probability of packet 1 also being a valid packet (i.e. the burst length $\beta$ of late packets is 0 ) is

$$
\operatorname{Pr}(\beta=0)=\operatorname{Pr}\left(Z_{1} \leq\lfloor\mu / \lambda\rfloor\right) .
$$

The probability of packet 1 being a late packet but packet 2 being a valid packet (i.e. the burst length of late packets is 1) is

$$
\operatorname{Pr}(\beta=1)=\operatorname{Pr}\left(Z_{1}>\lfloor\mu / \lambda\rfloor, Z_{1}+Z_{2} \leq\lfloor 2 \mu / \lambda\rfloor\right) .
$$

Likewise, the probability of having exactly $K$ consecutive late packets is

$$
\begin{aligned}
\operatorname{Pr}(\beta=K)= & \operatorname{Pr}\left(Z_{1}>\lfloor\mu / \lambda\rfloor,\right. \\
& Z_{1}+Z_{2}>\lfloor 2 \mu / \lambda\rfloor, \\
& \cdots, \\
& Z_{1}+Z_{2}+\cdots+Z_{K}>\lfloor K \mu / \lambda\rfloor, \\
& \left.Z_{1}+Z_{2}+\cdots+Z_{K+1} \leq\lfloor(K+1) \mu / \lambda\rfloor\right) .
\end{aligned}
$$

Substituting Equation (5) into Equation (9), we can obtain $\operatorname{Pr}(\beta=K)$ as shown on the top of the next page. Then the average burst length can be calculated as

$$
E[\beta]=\sum_{k=1}^{\infty} k \cdot \operatorname{Pr}(\beta=k) .
$$

Recall that the state of the system is reset with the transmission of a valid packet, so the system is modeled as a valid packet followed by a burst of $\beta$ late packets. We can thus derive the probability of late packets for 802.11 as

$$
p_{\text {late }}^{(802.11)}=\frac{E[\beta]}{E[\beta]+1} .
$$

Finally, combining (6) and (11), the overall loss rate for 802.11 's count-based retransmission strategy is

$$
p_{\text {loss }}^{(802.11)}=p_{\text {error }}^{(802.11)}+\left(1-p_{\text {error }}^{(802.11)}\right) p_{\text {late }}^{(802.11)} \text {. }
$$

Fig. 3 compares the overall loss rates of TAR and 802.11 for $\frac{\mu}{\lambda}=4$ and $P_{e}=0.75$. The figure shows a tradeoff between packet erasures and late arrivals in 802.11: a large retry limit mitigates packet erasures but it may create more late packets; on the other hand, a small retry limit reduces late arrivals but it is prone to errors. While a poor choice of retry limit can hurt the performance of an 802.11-like strategy, TAR automatically achieves the best retransmission strategy by adapting the number of retransmissions dynamically.

In practice it is hard to determine the optimal retry limit a priori because wireless channel conditions and video traffic characteristics change over time, requiring constant updating of the retry limit for each source-destination pair. TAR does not require the knowledge of underlying channel conditions nor traffic characteristics, making it a more promising solution than the count-based strategies.

\section{B. Analysis of Opportunistic Retransmission}

We now study the performance of PRO. For simplicity, the following analysis assumes zero-overhead and error-free feedback. With the assumption of a memoryless packet erasure channel, we can model opportunistic retransmission as a discrete-time Markov chain with time-homogeneous transition probabilities. Consider an $N$-node network with the source labeled as 0 , the destination labeled as $N-1$, and $N-2$ candidate relays labeled as $1,2, \cdots, N-2$. Let $P_{m n}$ denote the link error rate from node $m$ to node $n$. The system state $S=\left(\right.$ bin $\left.b_{N-1} b_{N-2} \cdots b_{1}\right)$, where $b_{i}=\{0,1\}$, is defined as an $(N-1)$-bit number with the $n$-th bit $b_{n}$ representing the packet reception status of node $n$ ( 1 is successful reception and 0 is a miss). For example, the four-node network in Fig. 1 contains a source (node 0 ), a destination (node 3 ), and two 


$$
\operatorname{Pr}(\beta=K)=\sum_{\substack{z_{1}=\left\lfloor\frac{\mu}{\lambda}\right\rfloor+1 \\ \min \left\{\left\lfloor(K+1) \frac{\mu}{\lambda}\right\rfloor\right.}}^{\left.R+\left(z_{2}+z_{2}+z_{2}+\cdots+z_{K}\right), R+1\right\}} \sum_{z_{K+1}=1}^{R+1} \cdots \sum_{z_{K}=\max \left\{\left\lfloor K \frac{\mu}{\lambda}\right\rfloor+1-\left(z_{1}+z_{2}+\cdots+z_{K-1}\right), 1\right\}}^{R+1}\left(s_{z_{1}}^{(802.11)} s_{z_{2}}^{(802.11)} \cdots s_{z_{K+1}}^{(802.11)}\right) .
$$

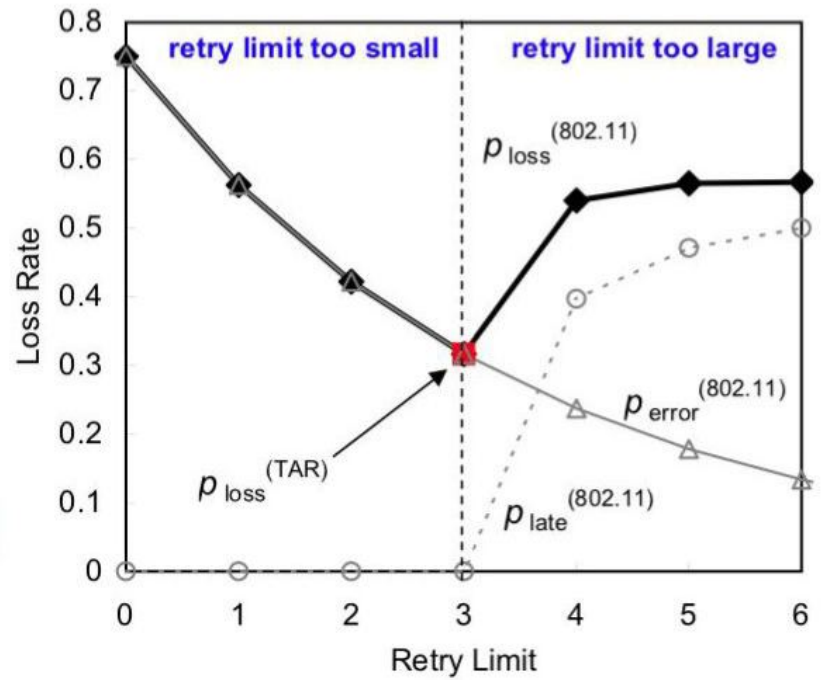

Fig. 3. Loss rate comparison of TAR and 802.11

relays (node 2 and node 3 ). State $1=$ (bin 001) represents node 1 has received the packet but node 2 and node 3 have not. State $2=$ (bin 010) represents node 2 has received the packet but node 1 and node 3 have not. States with the left-most bit $b_{N-1}$ set indicate successful deliveries to the destination and to simplify the model, they are grouped into one single state, state $2^{N-2}$. The resulting model is a $\left(2^{N-2}+1\right)$-state Markov chain.

The system starts at state 0 when the source is about to send a new packet. Every state transition is a (re)transmission of the packet. The (re)transmission process terminates at state $2^{N-2}$ which indicates that the destination has successfully received the packet. Hence the goal of this analysis is to find the expected number of state transitions going from the initial state 0 to the sink state $2^{N-2}$.

Let $\mathbf{A}=\left[a_{(i+1)(j+1)}\right]_{i=0,1, \cdots, 2^{N-2}: j=0,1, \cdots, 2^{N-2}}$ be the transition probability matrix in which $a_{(i+1)(j+1)}$ is the transition probability from state $i$ to state $j$. In the ideal case, the best relay for retransmitting a packet should be the one with the strongest connectivity to the destination among the nodes that currently have a copy of the packet. Without loss of generality, we assume nodes labeled with a larger number have a smaller link error rate to the destination (i.e. $\left.P_{0(N-1)} \geq P_{1(N-1)} \geq \cdots \geq P_{(N-2)(N-1)}\right)$. This means that the highest-numbered node out of the set of nodes that have a copy of the packet is the best relay to retransmit the packet, and we will assume that this is indeed the node that retransmits the packet. For a particular state, this is the node corresponding to the left-most 1 in the binary representation of the state. We can then write the transition probability $a_{(i+1)(j+1)}$ as the

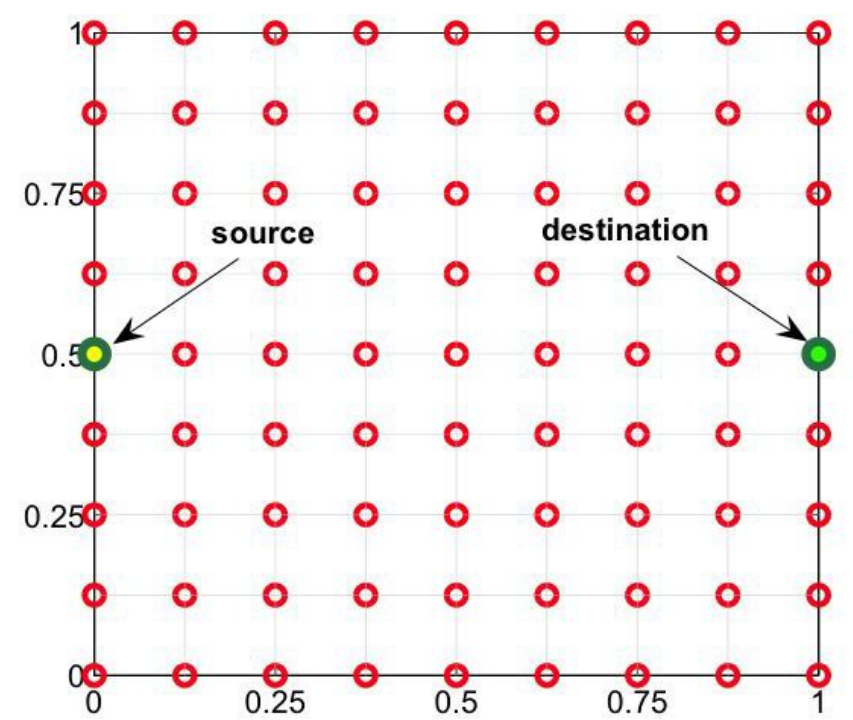

Fig. 4. Grid network topology with $\eta=8$.

probability that the packet reception status of all the nodes change from $i$ to $j$ after a transmission from the best relay in state $i$. As an example, the transition probability matrix of the four-node network is given in Equation (14).

Starting with the initial state probability $\pi^{(0)}=\left[\begin{array}{llll}1 & 0 & \cdots & 0\end{array}\right]$, we can then iteratively obtain the $k$-th step state probability as $\pi^{(k)}=\pi^{(0)} \mathbf{A}^{k}$. The $k$-th step state probability reveals the likelihood of all possible packet reception status for a packet after $k$ transmissions. We are interested in the probability of the sink state, i.e. state $2^{N-2}$, in the $k$-th step which corresponds to the probability of successfully delivering a packet with $k$ or fewer transmissions. Let $X$ be the random variable representing the number of transmissions needed to successfully deliver a packet. We can then write

$$
\pi_{2^{N-2}}^{(k)}=\operatorname{Pr}(X \leq k)
$$

which is the cumulative distribution function (CDF) of $X$. Thus the average number of transmissions needed to deliver a packet using opportunistic retransmission can be obtained as

$$
\begin{aligned}
E[X] & =\sum_{k=1}^{\infty} k \cdot(\operatorname{Pr}(X \leq k)-\operatorname{Pr}(X \leq k-1)) \\
& =\sum_{k=1}^{\infty} k \cdot\left(\pi_{2^{N-2}}^{(k)}-\pi_{2^{N-2}}^{(k-1)}\right)
\end{aligned}
$$

If we view the source and relays jointly as a sending system and the network as a transmission system that connects the sending system to the destination, the packet error rate

$$
P_{e}=1-\frac{1}{E[X]}
$$




$$
\mathbf{A}=\left[\begin{array}{ccccc}
\left(1-P_{01}\right)\left(1-P_{02}\right)\left(1-P_{03}\right) & P_{01}\left(1-P_{02}\right)\left(1-P_{03}\right) & \left(1-P_{01}\right) P_{02}\left(1-P_{03}\right) & P_{01} P_{02}\left(1-P_{03}\right) & P_{03} \\
0 & \left(1-P_{12}\right)\left(1-P_{13}\right) & 0 & P_{12}\left(1-P_{13}\right) & P_{13} \\
0 & 0 & \left(1-P_{21}\right)\left(1-P_{23}\right) & P_{21}\left(1-P_{23}\right) & P_{23} \\
0 & 0 & 0 & \left(1-P_{23}\right) & P_{23} \\
0 & 0 & 0 & 0 & 1
\end{array}\right]
$$

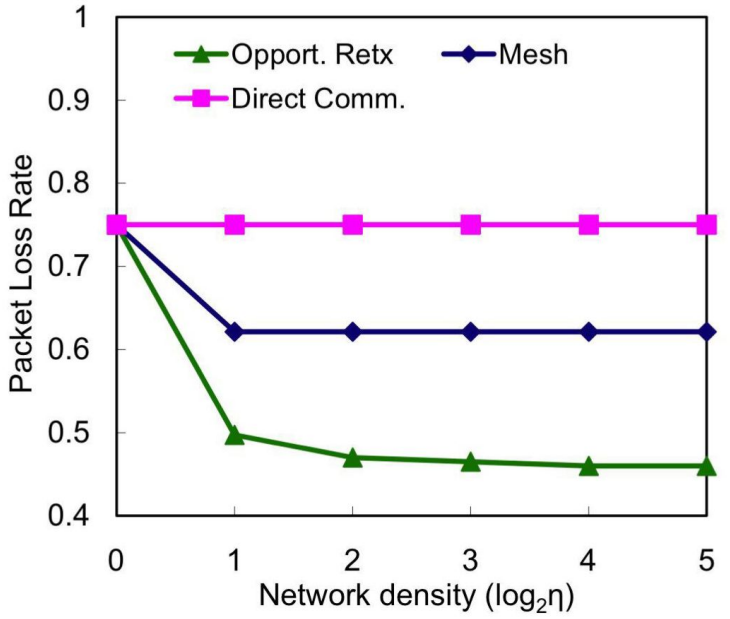

Fig. 5. Loss rate comparison of a $\eta \times \eta$ square grid topology with varied network densities (defined as $\log _{2} \eta$ )

can then be substituted into (12) to obtain the video transmission performance of PRO.

\section{Analysis of Hybrid Spatial/Temporal Retransmission}

By combining PRO and TAR, we can use opportunistic retransmission to specifically optimize video transmission by avoiding late packets. The performance of the resulting scheme, PROTAR can be obtained by substituting Equation (17) into Equation (4).

We use a regular square grid topology to study the analytical performance of PROTAR. The source and the destination nodes are the midpoints of the left and right edges of the grid, respectively. The distance between the source and the destination is fixed and we change the topology by changing the number of relays. Let $\eta$ be the number of segments on the line connecting the source and destination nodes. $\eta=1$ corresponds to the case of no relays, and increasing $\eta$ increases both the number and density of the relays. Except for the "no relay" case, we only consider even values for $\eta$ so the number of nodes in grid is $(\eta+1)^{2}$. Fig. 4 shows an example grid with $\eta=8$. The link error rate between nodes $m$ and $n$ is modeled as

$$
P_{m n}=1-\frac{\left(1-P_{s d}\right)}{d_{m n}^{1.6}}
$$

where $P_{s d}$ is the link error rate from the source to the destination and $d_{m n}$ is the distance between node $m$ and node $n$, normalized relative to the distance between the source and destination, i.e. $d_{s d}=1^{1}$. Fig. 5 shows the analytical results for grid topologies with different $\eta$. We use $P_{s d}=0.75$ and $\frac{\mu}{\lambda}=4$ for the traffic load. We use the default long retry limit

${ }^{1}$ Equation (18) approximates link error rates with a log-distance path loss with a path loss exponent of 1.6.
$(=4)$ in the 802.11 specification as the retry limit for the count-based strategy [20]. For completeness, the figure also includes results for the mesh network-based approach (Mesh) that uses the optimal multi-hop path between the source and the destination to forward packets. The results show that PROTAR can combine the gains of TAR and PRO to achieve the best performance.

\section{Vi. Performance Evaluation}

We have implemented PROTAR in FlexMAC [18], a MAC framework for wireless NICs based on the Atheros chipset. Video streaming is based on RTP over UDP [30]. PROTAR identifies video packets via the transport source and destination port numbers, which are pre-configured via an out-of-band scheme [32]. Newly-established RTP sessions are detected through the use of a new synchronization source identifier (SSRC) in the RTP header and PROTAR then uses the RTP timestamp field and knowledge of the startup buffer delay to calculate the retransmission deadline. Relays follow the procedure in Section IV-D to retrieve retransmission deadlines.

Unless otherwise notes, our experiments use video sequences encoded in MPEG-4 CIF format at 15 frames/second and 15 frames/GoP (Group of Pictures) with a quantization step size of 4 . The GoP format is IBBPBBPBBPBBPBB. We evaluate five transmission protocols: (1) 802.11, (2) TAR, (3) PRO, (4) PROTAR, and (5) Mesh. The 802.11 PHY offers a multi-rate capability that allows dynamic transmit rate adaptation to improve performance under different channel conditions [20]. In our experiments we use the rate adaptation algorithm described in [22] for 802.11 and TAR. PRO, Mesh and PROTAR always use the highest transmit rate because their philosophy is to leverage relays to aid in retransmission, rather than relying exclusively on the sources for recovery. The protocol parameters used in the evaluation are summarized in Table I.

\section{A. Objective Visual Tests}

We present objective test results for different transmission protocols in both a controlled testbed and the real world. The controlled experiments use the CMU wireless network emulator which supports realistic and fully controllable and repeatable wireless experiments [23]. The real world experiments are conducted in two buildings on the CMU campus, so these experiments automatically account for all effects that are naturally present in deployed wireless networks, e.g. interference, noise, multi-path fading, and shadowing. The results presented in this section were done using $802.11 \mathrm{~b}$ (instead of $802.11 \mathrm{~g}$ ) due to the limitation of timing precision of the emulator. However, the conclusion made in this section should be also applicable to $802.11 \mathrm{~g}$ networks as our protocols are largely independent of the underlying physical technology. 
TABLE II

OBJECTIVE VISUAL QUALITY RESULTS

(a) Testbed Results

\begin{tabular}{|c|c|c|c|}
\hline Technique & Late Pkts $(\%)$ & Avg. PSNR(dB) & Max Frz(sec) \\
\hline 802.11 & 17.0 & 23.9 & 3.6 \\
TAR & 1.1 & 27.1 & 0.9 \\
Mesh & 11.9 & 29.1 & 3.4 \\
PRO & 8.1 & 30.3 & 1.9 \\
PROTAR & 2.1 & 32.7 & 0.7 \\
\hline
\end{tabular}

(b) Real-world Results

\begin{tabular}{|c|c|c|c|}
\hline Technique & Late Pkts(\%) & Avg. PSNR(dB) & Max Frz(sec) \\
\hline 802.11 & 23.1 & 15.7 & 6.5 \\
TAR & 3.0 & 17.8 & 0.9 \\
Mesh & 15.5 & 20.1 & 4.9 \\
PRO & 13.8 & 22.5 & 3.1 \\
PROTAR & 2.9 & 25.0 & 0.9 \\
\hline
\end{tabular}

We used the stefan test video. For each packet, the retransmission deadline is set to the end of the associated GoP, i.e. all packets in the same GoP are assigned an equal retransmission deadline. This implicitly offers unequal error protection [13][15] because reference frames are transmitted before inter-coded frames in a GoP. With a FIFO queueing discipline, inter-coded frames will not be transmitted until after the successful deliveries of reference frames. The destination collects a packet trace that records the received packets together with a timestamp. The trace information is then used to calculate the distortion of the received video stream. We use a common error concealment scheme to deal with packets that are lost either due to erasures or late arrivals, namely missing blocks in the frame are copied from the last correctly decoded frame.

1) Emulator Testbed Results: The testbed scenario contains seven nodes: source, destination, and five relays. The distance between the source (video server) and the destination (video client) is initialized to 100 meters. Five relays are uniformly placed between the source and the destination (adjacent relays are spaced at 16.7 meters). The signal propagation model combines log distance attenuation with a path loss exponent of 2.8 with Ricean fading with $K=3$. The retry limit is 5 for the count-based retransmission techniques; we chose this limit because it results in the best PSNR for this set of experiments.

The experimental results are given in Table III(a). We see that compared with 802.11, TAR results in much fewer late arrivals, which leads to better video quality. In addition, the maximum playback freezing period is bounded by one GoP period $(<1$ second $)$ due to the assignment of equal retransmission deadlines for the packets in a GoP. Exploiting relays also increases PSNR performance relative to 802.11, as it increases throughput of the network, but reduction in late packets and maximum freeze time are lower than TAR since PRO does not specifically optimize latency requirements of video. The results also indicate that PRO outperforms Mesh, showing the benefit of opportunistically taking advantage of successful delivery via the direct path on a per packet basis. Overall, PROTAR achieves the best performance, by adding timing optimizations specific to video to PRO.

2) Real-world Results: In the real-world experiments, we use ten laptops (labeled as nodes 1 through 10) randomly placed on a single floor of an office building with hard partitions (see Fig. 6(a)). The experiments are conducted

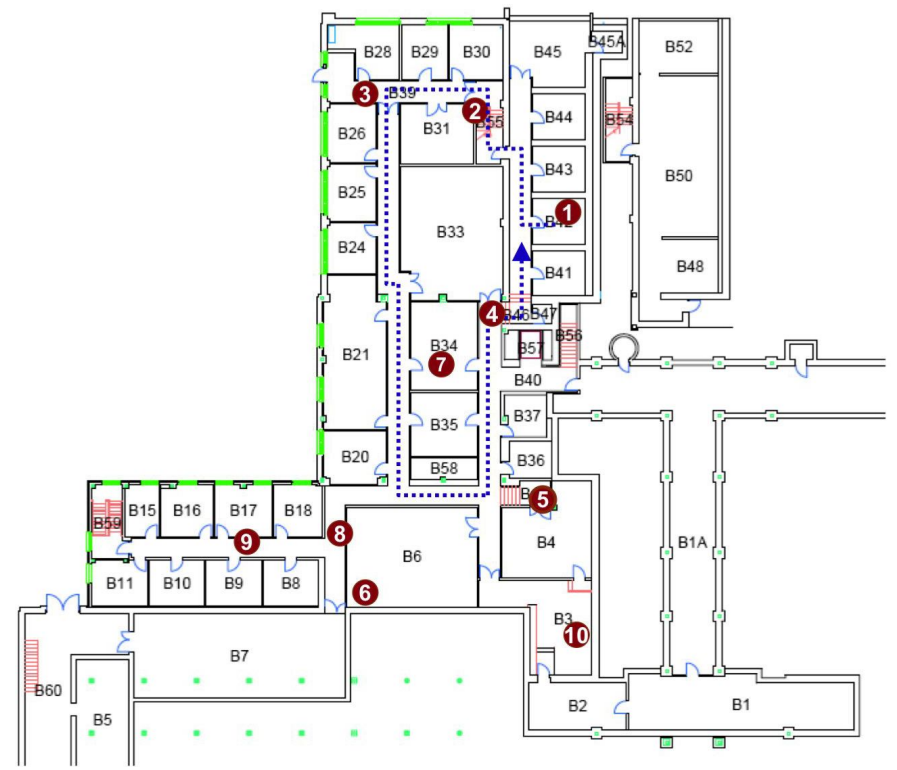

(a) Office building

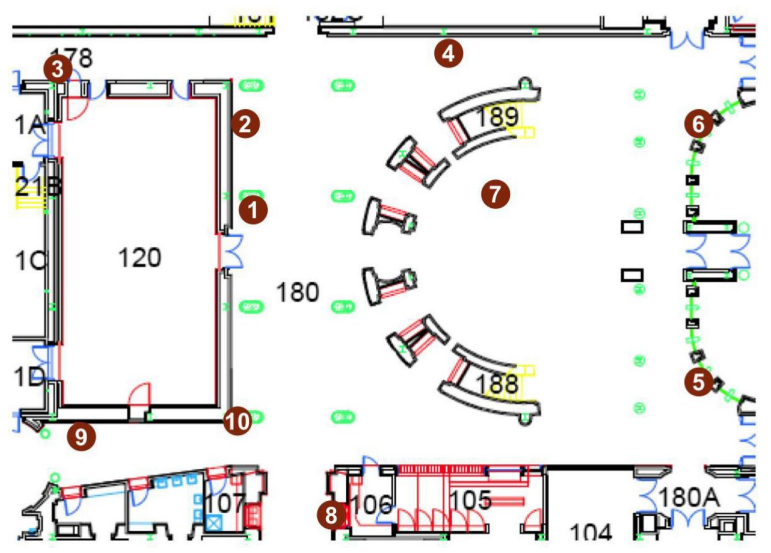

(b) Student lounge

Fig. 6. Test floor plan

during the night time, when changes in the environment are relatively limited. To further alleviate environmental variation across experiments, we average the performance results for three server-client pairs and we interleave the test order of the different techniques. The three server-client pairs are $(1,7)$, $(7,9)$ and $(10,4)$ (the left number is the video server and the right is the video client). For each experiment, nodes other than the server and the client may serve as relays. Table III(b) gives the experimental results. While the precise results are somewhat different from the testbed results, the relative performance of the five techniques in terms of late packets, average PSNR, and maximum freezing times are the same, so the same conclusions apply.

\section{B. Subjective Visual Tests}

To obtain subjective evaluation results, we set up visual experiments according to the CCIR Recommendation 5004 [24]. We conducted measurements using a public streaming server and media players for various realistic scenarios in the real world [25]. The video server and client are configured to support a 2-second startup buffer to smooth out delay jitter. 


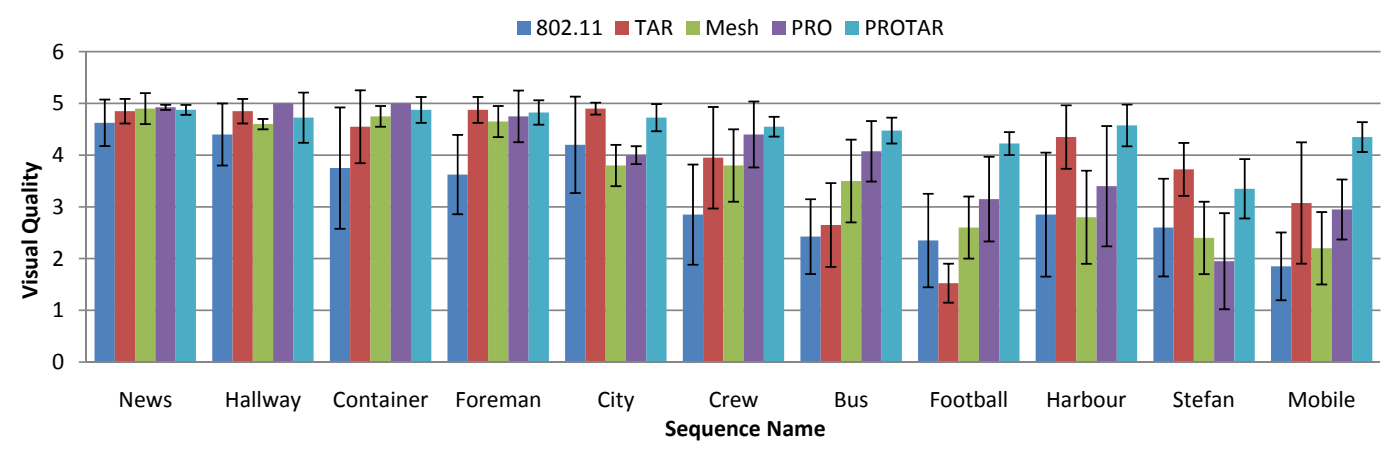

Fig. 7. Visual quality results for the single session scenario

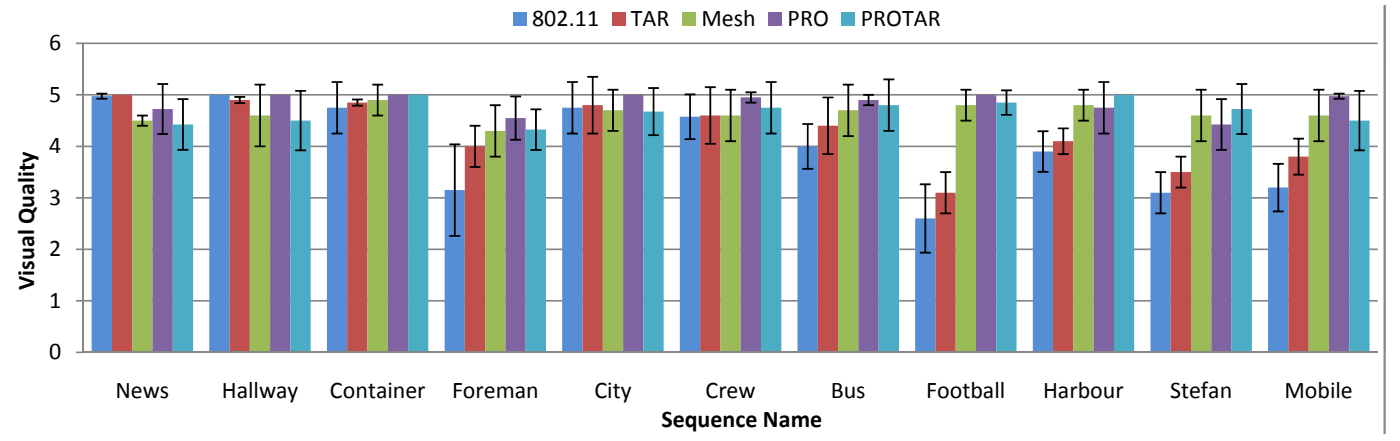

(a) Average visual quality of the highest-rated session

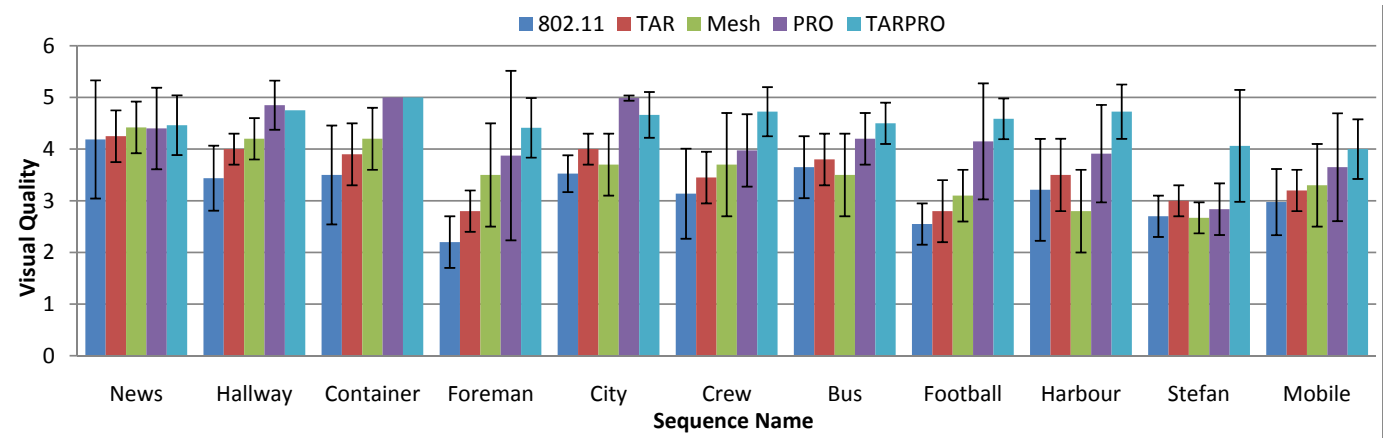

(b) Average visual quality of the lowest-rated session

Fig. 8. Visual quality results for the concurrent session scenario

TABLE III

ATTRIBUTES OF THE VISUAL QUALITY SCALE. THE FREEZING FREQUENCY IS DEFINED AS THE RATIO OF COMPLETELY FAILED FRAMES AND THE TOTAL FRAMES.

\begin{tabular}{|c|c|c|c|c|}
\hline Scale & Quality & Impairment & PSNR & Frz Freq \\
\hline 5 & Excellent & Imperceptible & 29.5 & 0 \\
4 & Good & Perceptible but not annoying & 27.8 & 0 \\
3 & Fair & Slight annoying & 23.6 & 0 \\
2 & Poor & Annoying & 20.6 & 0.25 \\
1 & Bad & Very annoying & 22.1 & 0.5 \\
\hline
\end{tabular}

Eleven test sequences are used to capture videos with different motion activities; Table IV summarizes the average bit rates for the sequences.

In this case the evaluation results consist of a visual quality metric assigned by human assessors for each video sequence transmitted using each of the protocols. The subjective visual quality assessment metric is a single number in the range from 1 to 5 , where 1 is the lowest perceived quality, and 5 is the highest. Before conducting the subjective tests, a calibration procedure is performed to mitigate perceptual variations among assessors: five video clips and their scores are shown to the assessors. The video clips are created with different degrees of artifacts (i.e. frame freezes, motion-jitter, blocking artifacts, and variation in visual quality). Table III shows the attributes of the five video clips that were used for callibration. During the evaluation, assessors first view the test sequences in the original CIF format and then assign a score for the transmitted sequences following the labeling principle explained in the calibration phase. Voting with a decimal fraction is allowed. Each test sequence is repeated to last 60 
TABLE IV

SUMMARY OF PERFORMANCE OVER DIFFERENT REAL-WORLD SCENARIOS

\begin{tabular}{|l|c||c|c|c|c|c|}
\hline & Video bit rate (quant. size 4) & 802.11 & TAR & Mesh & PRO & PROTAR \\
\hline News & $452 \mathrm{Kbps}$ & 3.8 & 4.4 & 4.3 & 4.3 & 4.6 \\
Hallway & $557 \mathrm{Kbps}$ & 3.4 & 4.0 & 4.2 & 4.7 & 4.7 \\
Container & $582 \mathrm{Kbps}$ & 3.2 & 4.1 & 4.5 & 4.9 & 4.9 \\
Foreman & $973 \mathrm{Kbps}$ & 2.8 & 3.5 & 3.9 & 4.3 & 4.5 \\
City & $993 \mathrm{Kbps}$ & 3.2 & 3.6 & 3.6 & 4.1 & 4.6 \\
Crew & $1.20 \mathrm{Mbps}$ & 2.6 & 3.0 & 3.4 & 3.8 & 4.3 \\
Bus & $1.85 \mathrm{Mbps}$ & 2.7 & 3.0 & 3.1 & 3.7 & 4.2 \\
Football & $1.91 \mathrm{Mbps}$ & 2.0 & 2.4 & 3.0 & 3.6 & 4.3 \\
Harbour & $1.92 \mathrm{Mbps}$ & 2.6 & 3.4 & 3.0 & 3.5 & 4.5 \\
Stefan & $2.45 \mathrm{Mbps}$ & 2.1 & 2.8 & 2.8 & 3.0 & 4.0 \\
Mobile & $2.80 \mathrm{Mbps}$ & 2.2 & 3.1 & 2.9 & 3.4 & 4.0 \\
\hline Average & & 2.8 & 3.4 & 3.5 & 3.9 & 4.4 \\
\hline
\end{tabular}

seconds. The transmission protocol used for each sequence is hidden from the assessors and the order of transmission techniques is randomized to avoid the expectation of a trend in the visual quality. Each sequence is labeled by five assessors and we present the average visual quality.

1) Single Session: In the first part of the evaluation we use the same setup as for the real-world experiments in the objective tests (see Section VI-A2). Fig. 7 shows the visual quality results when only one video session is active at a time. Each bar in the histogram shows the average and the standard deviation for 15 experiments corresponding to five assessors and three server-client pairs. In general, TAR outperforms 802.11, and PRO outperforms Mesh which outperforms 802.11. The relative performance difference between TAR and PRO is sequence dependent. PROTAR always outperforms the other strategies. The performance differences become more pronounced as the video bit rate increases. These results are generally consistent with the objective test results presented in Section VI-A.

2) Concurrent Sessions: Next we study a scenario with multiple concurrent sessions. The three server-client pairs used in the single session case are now running concurrently. To sustain multiple concurrent streams, test sequences with quantization step size 8 are used. The other coding parameters and experimental settings remain the same as in the single session case.

The two histograms in Fig. 8 show the average video quality and the standard deviation for the highest-rated and the lowestrated sessions. Similar to the single session results, TAR outperforms 802.11 and PRO outperforms Mesh. PROTAR generally has the best performance. In addition, we observe that the relay-based methods, PRO and Mesh, usually outperform the non-relay methods, TAR and 802.11, in this scenario. In other words, under heavy load, relaying turns out to be an effective way to deal with the fairly challenging clientserver channels. One reason is that 802.11 's rate adaptation algorithm occasionally misinterprets collisions as packet losses due to poor link quality and it erroneously reduces the transmit rate, which leads to an inefficient use of the channel [19]. In contrast, opportunistic or mesh-based relaying can use the highest transmit rate. Moreover, an equal channel access policy penalizes not just the channels that use a low transmit rate as a result of rate adaptation, but also flows with high transmit rates [26]. This affects the video quality for TAR and
802.11 in Fig. 8(a). Nevertheless, TAR is still useful although the achievable performance is limited by the aforementioned phenomenon.

3) Single Session with a Mobile Client: In this scenario, node 1 serves as the video server, streaming video data to a mobile client. The video client is moving along the hallway with a walking speed of about $1.5 \mathrm{~m} / \mathrm{sec}$. The trajectory is shown as the dashed line in Fig. 6(a). Fig. 9 shows the average visual quality result and the standard deviation. In contrast to the stationary scenarios, 802.11 performs very poorly for all the video sequences, including low-bit rate videos. The reason is that the video client sometimes passed through an out-ofreach region, where the client has trouble connecting to the video server (e.g. a spot near room B20 in Fig. 6(a)). During the out-of-reach period, the 802.11 MAC discards packets after reaching the retry limit so video playback freezes. However, with TAR, failed packets are persistently retried as long as the retransmission deadline has not elapsed. As a result, TAR performs significantly better than 802.11 . Not surprisingly, we observe that relays can improve communication with out-ofreach nodes. Moreover, the agility of PRO leads to better performance compared with Mesh. Again, PROTAR has the best overall performance.

4) Single Session in a Dynamic Environment: In our final scenario we ran experiments using ten laptops randomly placed in an open space student lounge (see Fig. 6(b)). The tests are conducted during the day time so a lot of students moving through the space, affecting the signal propagation environment. Again, we collected results for three server-client pairs, $(6,10),(8,6)$ and $(3,7)$ from five assessors. Fig. 10 shows the average visual quality result and the standard deviation. In comparison with the office building scenario, the performance benefits of PRO over TAR and 802.11 are more pronounced across all the test sequences, including low-bit rate videos. This suggests that PRO is very effective in dynamic environments. For low-bit rate videos, PRO sometimes outperforms PROTAR because the available network bandwidth can sustain excessive retransmissions reducing the benefits of TAR. Note that small differences in the results do not matter because of the limited number (five) of assessors. When relays are not available, the adoption of TAR is still helpful. Overall, PROTAR achieves the best performance.

Table IV summarizes the average visual quality results for all the above scenarios for each test sequence. The results 


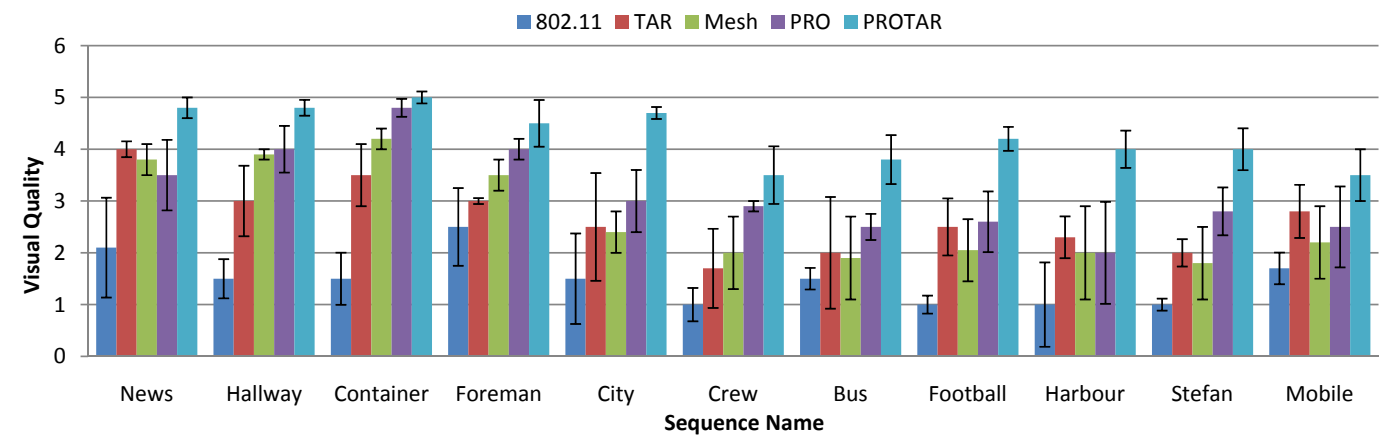

Fig. 9. Visual quality results for the mobile scenario

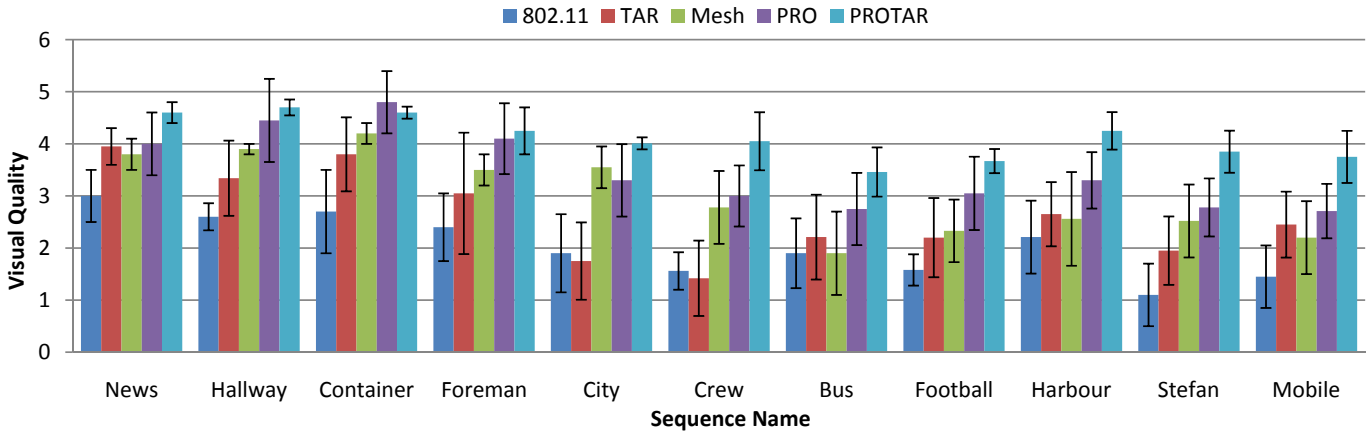

Fig. 10. Visual quality results for the single session scenario in a dynamic environment

clearly show that the hybrid scheme, PROTAR, that combines the benefits of PRO and TAR achieves the highest visual quality.

\section{CONCLUSION}

In this paper, we introduced hybrid spatial/temporal retransmission, building on our prior work of opportunistic retransmission (PRO) and time-based adaptive retransmission (TAR) to improve quality of video streaming services over wireless LANs. To increase individual throughput as well as overall network capacity, PRO relies on overhearing nodes to function as relays that retransmit failed packets on behalf of the source. Specifically, the best relay out of the set of nodes that currently have the copy of the packet is responsible for retransmitting (relaying) the packet. Relays with stronger connectivity to the destination have a higher chance of delivering packets successfully than the source, thus resulting in a more efficient use of the channel. To reduce the number of late arrivals, TAR uses a retransmission deadline rather than a fixed retry count. The gain of the proposed hybrid scheme is demonstrated using both theoretical analysis, and testbed and real-world experiments. Our extensive evaluation results demonstrate that the hybrid spatial/temporal retransmission scheme boosts video quality in a variety of wireless environments. The benefits are especially significant for high-contention channels, under fading, and for mobile users.

\section{REFERENCES}

[1] A. Majumdar, D.G. Sachs, I.V. Kozintsev, K. Ramchandran, and M. M. Yeung. Multicast and Unicast Real-Time Video Streaming over Wireless LANs, IEEE Trans. Circuits Syst. Video Technol., vol. 12, no. 6, pp. 524-534, June 2002.
[2] S.H. Kang and A. Zakhor, Packet scheduling algorithm for wireless video streaming, Packet Video Workshop 2002, April 2002.

[3] M. van der Schaar, S. Krishnamachari,S. Choi and X. Xu, Adaptive CrossLayer Protection Strategies for Robust Scalable Video Transmission Over 802.11 WLANs, IEEE J. Sel. Areas Commun. vol. 21. pp. 1752-1763, Dec. 2003.

[4] Q. Li and M. van der Schaar, Providing adaptive QoS to layered video over wireless local area networks through real-time retry limit adaptation, IEEE Trans. Multimedia, vol. 6, no. 2, pp. 278-290, 2004.

[5] G. Liebl, M. Kalman, B. Girod, Deadline-aware scheduling for wireless video streaming, IEEE International Conference on Multimedia and Expo (ICME), July 2005.

[6] H. Liu, W. Zhang, S. Yu, J. Cai, A Client-Driven Scalable Crosslayer Retransmission Scheme for $3 G$ Video Streaming, IEEE International Conference on Multimedia and Expo (ICME), July 2005.

[7] P. A. Chou and Z. Miao, Rate-Distortion Optimized Streaming of Packetized Media, IEEE Trans. Multimedia, Vol. 8, No. 2, April 2006.

[8] A. K. Miu, J. Apostolopoulos, W.-T. Tan and M. Trott, Low-Latency Wireless Video Over 802.11 Networks Using Path Diversity, IEEE International Conference on Multimedia and Expo (ICME), July 2003.

[9] R. Chakravorty, S. Banerjee and S. Ganguly, MobiStream: Error-resilient Video Streaming in Wireless WANs using Virtual Channels, IEEE Infocom, Barcelona, Spain, 2006.

[10] S. Ganesh Sitharaman and K. Maruthu Anantharaman, Impact of Retransmission Delays on Multilayer Video Streaming over IEEE 802.1le Wireless Networks, Proc. of the 2nd International Conference on COMmunication System softWAre and MiddlewaRE (COMSWARE 2007), Jan. 2007.

[11] P. Bucciol, E. Masala, E. Filippi, and J. C. DeMartin, Cross-Layer Perceptual ARQ for Video Communications over 802.11e Wireless Networks, Advances in Multimedia, Vol. 2007, Issue 1, Jan. 2007.

[12] D. Li, C.-N. Chuah, G. Cheung and S. J. B. Yoo, MUVIS: Multi-Source Video Streaming Service over WLANs, J. Communications and Networks, Special Issue on Towards the Next Generation Mobile Communications, vol. 7, no. 2, pp. 144-156, June 2005.

[13] M. H. Lu, P. Steenkiste, and T. Chen, Video Streaming over 802.11 WLAN with Content-aware Adaptive Retry, IEEE International Conference on Multimedia and Expo (ICME), July. 2005.

[14] M. H. Lu, P. Steenkiste, and T. Chen, Time-aware Oppourtunistic Relay for Video Streaming over WLANs, IEEE International Conference on Multimedia and Expo (ICME), July. 2007.

[15] M. Lu, P. Steenkiste and T. Chen, A Time-based Adaptive Retry Strategy for Video Streaming in 802.11 WLANs, Wireless Communications and 
Mobile Computing, Special Issue on Video Communications for $4 \mathrm{G}$ Wireless Systems, Jan. 2007.

[16] M. Lu, P. Steenkiste, and T. Chen, Video Transmission Over Wireless Multihop Networks Using Opportunistic Routing, Packet Video Workshop (PV2007), Nov. 2007.

[17] M. H. Lu, P. Steenkiste, and T. Chen, Design and Implementation of an Efficient Opportunistic Retransmission Protocol, The Fifteen International Conference on Mobile Computing and Networking (MobiCom'09), Sept. 2009.

[18] M. Lu, P. Steenkiste, and T. Chen, Using commodity hardware platform to develop and evaluate CSMA protocols, Proc. of ACM WiNTECH 2008, Sept. 2008.

[19] G. Judd, X. Wang, and P. Steenkiste, Efficient Channel-aware Rate Adaptation in Dynamic Environment, ACM Mobisys 2008, June 2008.

[20] Part 11: Wireless LAN Medium Access Control (MAC) and Physical Layer (PHY) Specifications, Ref. ISO/IEC 8802- 11:1999(E), IEEE Std. 802.11-199, 1999.

[21] IEEE. Wireless Medium Access Control (MAC) and Physical Lsyer (PHY) specification: Medium Access Control (MAC) Quality of Service (QoS) Enhancements.

[22] J.C. Bicket, Bit-rate Selection in Wireless Networks, Master's thesis, Massachusetts Institute of Technology, Feb. 2005.

[23] G. Judd and P. Steenkiste, Using Emulation to Understand and Improve Wireless Networks and Applications, Proc. of NSDI 2005.

[24] CCIR Recommendation 500-4, "Method for the Subjective Assessment of the Quality of Television Pictures," 1990.

[25] VLC media player, http://www.videolan.org/vlc/

[26] B. Sadeghi, V. Kanodia, A. Sabharwal, and E. Knightly, Opportunistic Media Access for Multirate Ad Hoc Networks, ACM MOBICOM, Sept. 2002.

[27] Z. Tao, K. Teo, J. Zhang, and T. Kuze, An Adaptive Frame Structure for OFDMA-based Mobile Multi-hop Relay Networks, IEEE C802.16j07/117, Jan. 2007, http://ieee802.org/16/relay/index.html.

[28] 3GPP Technical Report 25.848, version 4.0.0, Physical layer aspects of UTRA High Speed Downlink Packet Access, March 2001.

[29] D. L. Mills, RFC 1305 Network Time Protocol (Version 3) Specification, Implementation, March 1992.

[30] H. Schulzrinne, S. Casner, R. Frederick, and V. Jacobson, RFC 3550 RTP: A Transport Protocol for Real-Time Applications, July 2003.

[31] C. Perkins, E. Belding-Royer, and S. Das, RFC 3561 Ad hoc OnDemand Distance Vector (AODV) Routing, July 2003.

[32] G. Daugherty, Traversing Firewalls with Video over IP:Issues and Solutions (white paper), Visual Communications, Aug. 2003.

[33] S. Biswas and R. Morris ExOR: Opportunistic Multi-Hop Routing for Wireless Network, ACM SIGCOMM, Sept. 2005.

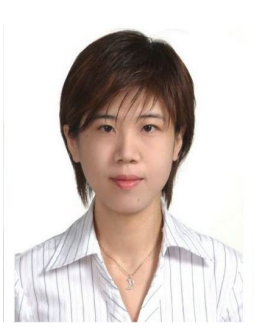

Mei-Hsuan Lu (S'04) received her B.A. degree in information management and M.S. degree in Computer Science and Information Engineering at National Taiwan University, Taiwan, in 1997 and 1999 and the M.S. degree and Ph.D. in Electrical and Computer Engineering from Carnegie Mellon University in 2008 and 2009. Her research interests span across video streaming, wireless networking, and mobile computing. From 1999 to 2003, she was a senior software engineer developing broadband communication and information appliance products at ASUSTeK Computer, Inc., Taiwan. In 2004, she served as a technical consultant in the broadband communication division at ASUS. During the summer of 2005, she was with HPLabs, Palo Alto, California, conducting research in adaptive video transcoding over 3GPP networks. She is a student member of the IEEE.

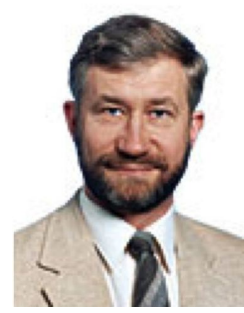

Peter Steenkiste (F'09) is a professor of Computer Science and of Electrical and Computer Engineering at Carnegie Mellon University. His research interests are in the areas of networking and distributed systems. He has done research in highperformance networking and distributed computing, network quality of service, overlay networking, and autonomic computing. His current research is the areas of wireless networking, pervasive computing, and self-management network services. He has an engineering degree from the University of Gent, Belgium and an M.S. degree and Ph.D. in Electrical Engineering from Stanford University. He is a fellow of the IEEE and a member of the ACM. His web page is at http://www.cs.cmu.edu/ prs.

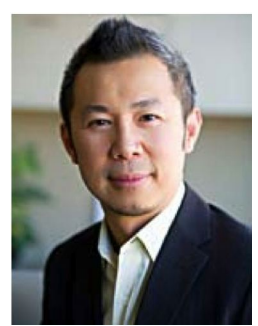

Tsuhan Chen (F'07) received the B.S. degree in electrical engineering from the National Taiwan University, Taipei, in 1987 and the M.S. and Ph.D. degrees in electrical engineering from the California Institute of Technology, Pasadena, in 1990 and 1993, respectively. He has been with the School of Electrical and Computer Engineering, Cornell University, Ithaca, New York, since January 2009, where he is Professor and Director. From October 1997 to December 2008, he was with the Department of Electrical and Computer Engineering, Carnegie Mellon University, Pittsburgh, PA, as Professor and Associate Department Head. From August 1993 to October 1997, he worked at AT\&T Bell Laboratories, Holmdel, NJ. He coedited a book titled Multimedia Systems, Standards, and Networks (Marcel Dekker, 2000).

Prof. Chen served as the Editor-in-Chief for the IEEE TRANSACTIONS ON Multimedia in 2002-2004. He also served in the Editorial Board of IEEE Signal Processing Magazine and as Associate Editor for IEEE TRANSACTIONS ON CIRCUITS AND SYSTEMS FOR VIDEO TECHNOLOGY, IEEE TRans actions on IMAGE Processing, IEEE Transactions on Signal Processing, and IEEE Transactions on Multimedia. He received the Charles Wilts Prize at the California Institute of Technology in 1993. He was a recipient of the National Science Foundation CAREER Award, from 2000 to 2003. He received the Benjamin Richard Teare Teaching Award in 2006, and the Eta Kappa Nu Award for Outstanding Faculty Teaching in 2007. He was elected to the Board of Governors, IEEE Signal Processing Society, 2007-2009, and a Distinguished Lecturer, IEEE Signal Processing Society, 2007-2008. He is a member of the Phi Tau Phi Scholastic Honor Society. 